Bull. Austral. Math. Soc.

VoL. $74(2006)$ [121-132]

\title{
B.H. NEUMANN'S QUESTION ON ENSURING COMMUTATIVITY OF FINITE GROUPS
}

\author{
A. Abdollahi, A. Azad, A. Mohammadi Hassanabadi and M. Zarrin
}

\section{Dedicated to the memory of Bernhard H. Neumann}

This paper is an attempt to provide a partial answer to the following question put forward by Bernhard H. Neumann in 2000: "Let $G$ be a finite group of order $g$ and assume that however a set $M$ of $m$ elements and a set $N$ of $n$ elements of the group is chosen, at least one element of $M$ commutes with at least one element of $N$. What relations between $g, m, n$ guarantee that $G$ is Abelian?" We find an exponential function $f(m, n)$ such that every such group $G$ is Abelian whenever $|G|>f(m, n)$ and this function can be taken to be polynomial if $G$ is not soluble. We give an upper bound in terms of $m$ and $n$ for the solubility length of $G$, if $G$ is soluble.

\section{INTRODUCTION AND RESULTS}

This paper is an attempt to provide a partial answer to the following question put forward by Bernhard H. Neumann in [10]: "Let $G$ be a finite group of order $g$ and assume that however a set $M$ of $m$ elements and a set $N$ of $n$ elements of the group is chosen, at least one element of $M$ commutes with at least one element of $N$ (call this condition Comm $(m, n)$ ). What relations between $g, m, n$ guarantee that $G$ is Abelian?"

Following Neumann, for given positive integers $m$ and $n$ we say that a group $G$ satisfies the condition $\operatorname{Comm}(m, n)$ if and only if for every two subsets $M$ and $N$ of cardinalities $m$ and $n$ respectively, there are elements $x \in M$ and $y \in N$ such that $x y=y x$.

We note that an infinite group $G$ satisfying the condition $\operatorname{Comm}(m, n)$ for some $m$ and $n$ is Abelian. This is because every infinite subset of such group contains two commuting elements. Thus by a famous Theorem of Neumann [9], it is centre-by-finite. Therefore $Z(G)$, the centre of $G$ is infinite. Now let $M$ and $N$ be two subsets of $Z(G)$, of sizes $m$ and $n$ respectively. Then for any two elements $x$ and $y$ of $G$, there are elements $z_{1} \in M$ and $z_{2} \in N$ such that $x z_{1} y z_{2}=y z_{2} x z_{1}$, so that $x y=y x$; namely $G$ is

Received 21st March, 2006

This work was in part supported by the Center of Excellence for Mathematics, University of Isfahan.

Copyright Clearance Centre, Inc. Serial-fee code: 0004-9727/06 \$A2.00+0.00. 
Abelian. Therefore in considering non-Abelian groups satisfying $\operatorname{Comm}(m, n)$ we need only consider finite cases.

We use the usual notations: for example $C_{G}(a)$ is the centraliser of an element $a$ in a group $G, S_{n}$ is the symmetric group on $n$ letters, $A_{n}$ is the alternating group on $n$ letters, $D_{2 n}$ is the dihedral group of order $2 n, Q_{8}$ is the quaternion group of order 8 and $T$ will stand for the group $\left\langle x, y \mid x^{6}=1, y^{2}=x^{3}, y^{-1} x y=x^{-1}\right\rangle$. If $G$ satisfies the condition $\operatorname{Comm}(m, n)$, then we say $G$ is a $C(m, n)$-group, or $G \in C(m, n)$.

Throughout $G$ will denote a finite non-Abelian group unless otherwise is stated. We shall show that a $C(m, n)$-group has order bounded by a function of $m$ and $n$ which may not always be chosen to be a polynomial function in terms of $m$ and $n$. Our main results are

ThEOREM 1.1. Let $G$ be a $C(m, n)$-group. Then $|G|$ is bounded by a function of $m$ and $n$.

The solubility length of a soluble $C(m, n)$-group is bounded above in terms of $m$ and $n$. In fact we prove the following.

ThEOREM 1.2. Let $G \in C(m, n)$ be a soluble group of solubility length $d$. Then

$$
d \leqslant \max \left\{\left\lceil\log _{2} m\right\rceil,\left\lceil\log _{2} n\right\rceil\right\}
$$

We also obtain a solubility criterion for $C(m, n)$-groups in terms of $m$ and $n$, namely

TheOREM 1.3. Let $G$ be a $C(m, n)$-group and $m+n \leqslant 58$. Then $G$ is a soluble group.

We give a complete characterisation of $C(m, n)$-groups, where $m+n \leqslant 10$, in the next theorem.

THEOREM 1.4. Let $G$ be a $C(m, n)$-group, where $m+n \leqslant 10$. Then $G$ is isomorphic to one of the following: $S_{3}, D_{2 n}$ for $n \in\{3,4,5,6\}, Q_{8}, T$ or a non-Abelian group of order 16 whose centre is of order 4 .

\section{A PARTIAL ANSWER TO NeUMANN'S QUESTION}

A subset of a non-Abelian group $G$ no two of whose distinct elements commute is called non-commuting. A non-commuting subset of maximal size is called a maximal non-commuting set and this maximal size will be denoted by $\omega(G)$. In this section we give a partial answer to Neumann's question by proving that a $C(m, n)$-group has the order bounded by a function of $m$ and $n$.

Proof of Theorem 1.1: Let $Z(G)=\left\{z_{1}, z_{2}, \ldots, z_{t}\right\}$, where $t \geqslant \max \{m, n\}$. Choose any two elements $a$ and $b$ in $G$, and put

$$
M=\left\{a z_{1}, a z_{2}, \ldots, a z_{m}\right\} \text { and } N=\left\{b z_{1}, b z_{2}, \ldots, b z_{n}\right\}
$$


Since $G$ is a $C(m, n)$-group, there exist $a z_{i} \in M$ and $b z_{j} \in N$, where $1 \leqslant i \leqslant m$ and $1 \leqslant i \leqslant n$, such that $a z_{i} b z_{j}=b z_{j} a z_{i}$. This implies that $a b=b a$, and so $G$ is an Abelian group, which is a contradiction. Thus $|Z(G)|<\max \{m, n\}$. Suppose, for a contradiction, that $\omega=\omega(G) \geqslant m+n$. Then there are $\omega$ pairwise non-commuting elements $a_{1}, \ldots, a_{m+n} \in G$. Put

$$
M=\left\{a_{1}, \ldots, a_{m}\right\} \text { and } N=\left\{a_{m+1}, \ldots, a_{m+n}\right\}
$$

Since $G$ is a $C(m, n)$-group, there exist $a_{i} \in M$ and $a_{j} \in N$ such that $a_{i} a_{j}=a_{j} a_{i}$, which is a contradiction. Thus $\omega<m+n$. Now the main result of [11] implies that $|G: Z(G)| \leqslant c^{\omega}$, where $c$ is a constant. Therefore

$$
|G| \leqslant c^{\omega}|Z(G)| \leqslant c^{m+n} \max \{m, n\},
$$

which completes the proof.

REMARK 2.1. Since an extra-special 2-group of order $2^{2 k+1}$, has maximal non-commuting sets of size $2 k+1$ (see [4] or [11]), if $f(m, n)$ is the least integer such that $|G| \leqslant f(m, n)$ for all $C(m, n)$-groups, then $f(m, n)$ cannot be chosen to be a polynomial in terms of $m$ and $n$.

The following is a key lemma to some of our results.

LEMma 2.2. Let $G$ be a $C(m, n)$-group and let $N$ be a normal subgroup of $G$ such that $G / N$ is non-Abelian. Then $|N|<\max \{m, n\}$.

Proof: Suppose on the contrary that $N=\left\{a_{1}, a_{2}, \ldots, a_{t}\right\}$ and $t \geqslant \max \{m, n\}$. Choose any two elements $x$ and $y$ in $G \backslash N$, and put

$$
\begin{aligned}
& X=\left\{x a_{1}, x a_{2}, \ldots, x a_{m}\right\} \text { and } \\
& Y=\left\{y a_{1}, y a_{2}, \ldots, y a_{n}\right\} .
\end{aligned}
$$

Since $G$ is a $C(m, n)$-group, there exist $x a_{i}$ in $X$ and $y a_{j}$ in $Y$ such that $\left[x a_{i}, y a_{j}\right]=1$. Thus $[x, y] \in N$ and $G / N$ is Abelian, which is a contradiction.

Corollary 2.3. Let $G$ be an insoluble $C(m, n)$-group. Then

$$
|G| \leqslant 4^{4}(m+n)^{8} \cdot \max \{m, n\} .
$$

Proof: Let $S$ be the largest soluble normal subgroup of $G$. Then $G / S$ has no non-trivial normal Abelian subgroup and by [12, Theorem 1.3], $|G / S|<(n(G))^{4}$, where $n(G)$ is the size of the largest conjugacy class in $G$. Now by [11] we have $n(G) \leqslant 4 \omega(G)^{2}$. Then by the proof of Theorem 1.1, $\omega(G)<m+n$ and by Lemma $2.2,|S|<\max \{m, n\}$, which completes the proof. 


\section{Soluble groups SATISFying the CONDition $\operatorname{Comm}(m, n)$}

In this section we prove Theorems 1.2 and 1.3. First we need some preliminary lemmas.

LEMMA 3.1. Let $G$ be a $C(m, n)$-group. If $a_{1}, a_{2}, \ldots, a_{n}$ are $n$ distinct elements of $G$, then $\left|G \backslash \bigcup_{i=1}^{n} C_{G}\left(a_{i}\right)\right|<m$.

Proof: Suppose, for a contradiction, that there exist $m$ distinct elements $b_{1}, b_{2}, \ldots, b_{m}$ in $G \backslash \bigcup_{i=1}^{n} C_{G}\left(a_{i}\right)$. Since $G$ is a $C(m, n)$-group, there exist elements $a_{i}, b_{j}$ such that $a_{i} b_{j}=b_{j} a_{i}$ and so $b_{j} \in C_{G}\left(a_{i}\right)$, which is a contradiction.

LEMMA 3.2. If $G$ is a $C(m, n)$-group, then $m+n \geqslant 6$.

Proof: Suppose, for a contradiction, that $m+n<6$. We distinguish two cases: CASE 1: $n=1$. Then $|G| \leqslant 6$ and so $G \cong S_{3}$, since $G$ is non-Abelian. If $a \in S_{3}$ is of order 3 , then Lemma 3.1 gives $3=\left|G \backslash C_{G}(a)\right|<m$. It follows that $m=4$. But $S_{3}$ is not a $C(1,4)$-group.

CASE 2: $\quad n=2$. Since $G$ is non-Abelian, there exists an element $a$ in $G \backslash Z(G)$ such that $a^{2} \neq 1$; for let $g^{2}=1$ for all $g \in G \backslash Z(G)$. Then $(g z)^{2}=1$ for all $z \in Z(G)$ and $g \in G \backslash Z(G)$. It follows that $1=g^{2} z^{2}=z^{2}$ and so we have $z^{2}=1$ for all $z \in Z(G)$. Hence $g^{2}=1$ for all $g \in G$ which implies that $G$ is Abelian, a contradiction.

Now since $a \neq a^{-1}$, it follows from Lemma 3.1 that

$$
\left|G \backslash\left(C_{G}(a) \cup C_{G}\left(a^{-1}\right)\right)\right| \leqslant m-1 \leqslant 2 .
$$

Since $C_{G}(a)=C_{G}\left(a^{-1}\right)$, we have that $|G| \leqslant\left|C_{G}(a)\right|+2$. As $a \in G \backslash Z(G)$, it follows that $\left|C_{G}(a)\right| \leqslant|G| / 2$ and so $|G| \leqslant|G| / 2+2$. Hence $|G| \leqslant 4$, so $G$ is Abelian. This contradiction completes the proof.

LEMMA 3.3. Let $G$ be a $C(m, n)$-group and let $N$ be a non-trivial normal subgroup of $G$. Then $G / N$ is a $C(m-r, n-t)$-group, for all positive integers $r, t$ such that $2 r \leqslant m$ and $2 t \leqslant n$.

Proof: Suppose, for a contradiction, that $G / N$ is not a $C(m-r, n-t)$-group. Thus there exist two subsets

$$
X=\left\{x_{1} N, \ldots, x_{m-r} N\right\} \text { and } Y=\left\{y_{1} N, \ldots, y_{n-t} N\right\}
$$

such that $\left[x_{i}, y_{j}\right] \notin N$ for all $i, j$. Let $a$ be a non-trivial element of $N$ and consider

$$
\begin{aligned}
X_{1} & =\left\{a x_{1}, \ldots, a x_{m-r}, x_{1}, \ldots, x_{r}\right\} \text { and } \\
Y_{1} & =\left\{a y_{1}, \ldots, a y_{n-t}, y_{1}, \ldots, y_{t}\right\}
\end{aligned}
$$


It is clear that $|X|=m$ and $|Y|=n$ and no element of $X_{1}$ commutes with no element of $Y_{1}$, which completes the proof.

Proof of Theorem 1.2: We argue by induction on $d$. By hypothesis $G$ is nonAbelian, thus it follows from Lemma 3.2 that either $m \geqslant 3$ or $n \geqslant 3$. Thus for $d=2$, the result holds, since $\left\lceil\log _{2} 3\right\rceil=2$. So assume that $d \geqslant 3$ and the result holds for $d-1$. Now $G / G^{(d-1)}$ has solubility length $d-1$. Let $k$ and $\ell$ be positive integers such that $2^{k}<m \leqslant 2^{k+1}$ and $2^{\ell}<n \leqslant 2^{\ell+1}$. Thus by Lemma 3.3, $G / G^{(d-1)}$ satisfies $\operatorname{Comm}\left(2^{k}, 2^{\ell}\right)$. Thus by the induction hypothesis $d-1 \leqslant \max \{k, \ell\}$ and so $d \leqslant \max \left\{\left\lceil\log _{2} m\right\rceil,\left\{\log _{2} n\right\rceil\right\}$, as required.

To prove Theorem 1.3 we need the following lemma.

If $G$ is a finite group, then for each prime divisor $p$ of $|G|$, we denote by $\nu_{p}(G)$ the number of Sylow $p$-subgroups of $G$.

LEMMA 3.4. Let $G$ be a $C(m, n)$-group and $p$ be a prime number dividing $|G|$ such that every two distinct Sylow p-subgroups of $G$ have trivial intersection. Then $\nu_{p}(G) \leqslant m+n-1$.

PROoF: It follows from the proof of Theorem 1.1, that $\omega(G)<m+n$. Now [7, Lemma 3] completes the proof.

Proof of ThEOREM 1.3: Suppose, on the contrary, that there exists a non-soluble finite group $G \in C(m, n)$ of the least possible order, where $m+n \leqslant 58$. If there exists a non-trivial proper normal subgroup $N$ of $G$, then both $N$ and $G / N$ are in $C(m, n)$ and so they are soluble. It follows that $G$ is soluble, which is a contradiction. Therefore $G$ is a minimal simple $C(m, n)$-group. By Thompson's classification of minimal simple groups [13], $G$ is isomorphic to one of the following simple groups:

$A_{5}$ the alternating group of degree 5 ,

$\operatorname{PSL}\left(2,2^{p}\right)$, where $p$ is an odd prime,

$\operatorname{PSL}\left(2,3^{p}\right)$, where $p$ is an odd prime,

$P S L(2, p)$, where $5<p$ is prime and $p \equiv 2(\bmod 5)$,

$\operatorname{PSL}(3,3)$, and

$S z\left(2^{p}\right), p$ an odd prime.

We first prove that $A_{5}$ is not a $C(m, n)$-group, where $m+n \leqslant 58$. Let $P_{1}, \ldots, P_{5}$, $Q_{1}, \ldots, Q_{10}, R_{1}, \ldots, R_{6}$ be Sylow $p$-subgroups of $A_{5}$, for $p=2,3,5$, respectively. It is easy to see that $A_{5}$ is the union of these Sylow subgroups and no two distinct non-trivial elements of coprime orders in $A_{5}$ commute (see [3]). Since every non-trivial element in $\bigcup_{i=1}^{6} R_{i} \cup Q_{1} \cup Q_{2}$ does not commute with one in

$$
\left(\bigcup_{i=1}^{5} P_{i} \cup \bigcup_{i=3}^{10} Q_{i}\right) \backslash\{a\}
$$


(where $a$ is an arbitrary non-trivial element of $\left.Q_{10}\right), A_{5}$ is not a $C(28,30)$-group and since every non-trivial element in

$$
\left(\bigcup_{i=1}^{6} R_{i} \cup Q_{1} \cup Q_{2} \cup Q_{3}\right) \backslash\{b\}
$$

(where $b$ is an arbitrary non-trivial element of $Q_{1}$ ) does not commute with one in

$$
\bigcup_{i=1}^{5} P_{i} \cup \bigcup_{i=4}^{10} Q_{i}
$$

$A_{5}$ is not a $C(29,29)$-group. Now suppose that $n \leqslant 27$. Then $n=4 k+\ell$ for some integers $k$ and $\ell$, where $0 \leqslant k \leqslant 6$ and $0 \leqslant \ell \leqslant 3$. Let $a$ be an arbitrary non-trivial element of $Q_{10}$ and define

$$
\mathcal{A}_{n}= \begin{cases}\bigcup_{i=1}^{k} R_{i} & \text { if } \ell=0 \\ \left(\bigcup_{i=1}^{k} R_{i} \cup Q_{10}\right) \backslash\{a\} & \text { if } \ell=1 \\ \bigcup_{i=1}^{k} R_{i} \cup Q_{1} & \text { if } \ell=2 \\ \bigcup_{i=1}^{k} R_{i} \cup P_{1} & \text { if } \ell=3\end{cases}
$$

and

$$
\mathcal{B}_{n}= \begin{cases}\left(\bigcup_{i=k+1}^{6} R_{i} \cup \bigcup_{i=1}^{5} P_{i} \cup \bigcup_{i=1}^{10} Q_{i}\right) \backslash\{a\} & \text { if } \ell=0 \\ \bigcup_{i=k+1}^{6} R_{i} \cup \bigcup_{i=1}^{5} P_{i} \cup \bigcup_{i=1}^{9} Q_{i} & \text { if } \ell=1 \\ \left(\bigcup_{i=k+1}^{6} R_{i} \cup \bigcup_{i=2}^{5} P_{i} \cup \bigcup_{i=2}^{10} Q_{i}\right) \backslash\{a\} & \text { if } \ell=2 \\ \left(\bigcup_{i=k+1}^{6} R_{i} \cup \bigcup_{i=2}^{5} P_{i} \cup \bigcup_{i=1}^{10} Q_{i}\right) \backslash\{a\} & \text { if } \ell=3\end{cases}
$$

Then no non-trivial element of $\mathcal{A}_{n}$ commutes with one of $\mathcal{B}_{n}$. It then follows that $A_{5}$ is not a $C(n, m)$-group, where $n+m \leqslant 58$.

If $G$ is isomorphic to $\operatorname{PSL}\left(2,2^{p}\right)$ or $\operatorname{PSL}\left(2,3^{p}\right)$, where $p$ is an odd prime, then by $[1$, Lemma 4.4], $\omega(G)>64$, which is a contradiction. If $G \cong P S L(3,3)$, then $|G|$ $=2^{4} \times 3^{3} \times 13$ so that $\nu_{13}(H)=144>57$, which is not possible by Lemma 3.4 . If $G \cong P S L(2, p)$ and $p>7$ ( $p$ is a prime number), then [1, Lemma 4.4] implies that $\omega(G) \geqslant 133$, a contradiction. If $G \cong P S L(2,7)$, then by [1, Proposition 3.21] and a similar argument as for $A_{5}$ we conclude that $G$ is not a $C(m, n)$-group. If $G \cong S z\left(2^{p}\right)$, then $|G|=2^{2 p} \times\left(2^{p}-1\right) \times\left(2^{2 p}+1\right)$ and $\nu_{2}(G)=2^{2 p}+1 \geqslant 65$ (see Theorem 3.10 (and its proof) of $[8$, Chapter XI]).

We note that the bound 58 in Theorem 1.3 is the best possible. In fact we have 
ThEOREM 3.5. The alternating group $A_{5}$ is the only non-Abelian finite simple $C(m, n)$-group, for some positive integers $m$ and $n$ such that $m+n=59$.

Proof: First we note that, since every centraliser of $A_{5}$ has order at least $3, A_{5}$ is a $C(1,58)$-group. For uniqueness, suppose, on the contrary, that there exists a non-Abelian finite simple group not isomorphic to $A_{5}$ and of least possible order which is a $C(m, n)$ group, for some positive integers $m$ and $n$ with $m+n=59$. Then by [5, Proposition 3], $G$ is isomorphic to one of the following groups:

$P S L\left(2,2^{p}\right), p=4$ or a prime;

$P S L\left(2,3^{p}\right), P S L\left(2,5^{p}\right), p$ a prime;

$P S L(2, p), p$ a prime and $7 \leqslant p$;

$P S L(3,3)$;

$P S L(2,5)$;

$P S U(3,4)$ (the projective special unitary group of degree 3 over the finite field of order $4^{2}$ ) or

$S z\left(2^{p}\right), p$ an odd prime.

Now an argument similar to the one in the proof of Theorem 1.3 gives a contradiction in each case.

4. Groups Satisfying the Condition $\operatorname{Comm}(m, n)$ for some small positive INTEGERS $m$ AND $n$

In this section we characterise $C(m, n)$-groups for some particular $m$ and $n$ and hence prove Theorem 1.4. First we need some preliminary lemmas.

Lemma 4.1. Let $G$ be a $C(m, n)$-group. Let $x$ be a non-central element of finite order such that $\varphi(|x|) \geqslant n$, where $\varphi$ is the Euler $\varphi$-function. Then $\left|G \backslash C_{G}(x)\right|<m$.

Proof: Suppose that

$$
\{k \in \mathbb{N}: 1 \leqslant k \leqslant|x| \text { and } \operatorname{gcd}(k,|x|)=1\}=\left\{d_{1}, d_{2}, \ldots, d_{\varphi(|x|)}\right\} .
$$

Since $x^{d_{i}} \neq x^{d_{j}}$ for all $i \neq j$, by Lemma 3.1

$$
\left|G \backslash \bigcup_{i=1}^{d_{\varphi(|z|)}} C_{G}\left(x^{d_{i}}\right)\right|<m .
$$

Also we have $C_{G}(x)=C_{G}\left(x^{d_{i}}\right)$ for all $1 \leqslant i \leqslant d_{\varphi(|x|)}$. Hence $\left|G \backslash C_{G}(x)\right|<m$.

Lemma 4.2. Let $G$ be a finite nilpotent $C(m, n)$-group. Then $\prod_{p \| G \mid} p<\max \{m, n\}$. 
Proof: The group $G$ is the direct product of its Sylow subgroups. So $G=\prod_{p \| G \mid} P$, where $P$ is the Sylow $p$-subgroup. Then $Z(G)=\prod_{p|| G \mid} Z(P)$ and $\max \{m, n\} \geqslant|Z(G)|$ $\geqslant \prod_{p|| G \mid} p$, by the proof of Theorem 1.1 .

LEMma 4.3. If $G$ is a $C(m, n)$-group, then for any prime divisor $p$ of $|G|$, $p \leqslant \max \{m, n\}$.

Proof: Suppose that $p$ is a prime divisor of $|G|$. Let $a$ be an element of order $p$ in $G$. For any $x$ in $G$ put $X=\left\{x a, x a^{2}, \ldots, x a^{m}\right\}$ and $Y=\left\{a, a^{2}, \ldots, a^{n}\right\}$. Then, by the hypothesis, there exist $x a^{i} \in X$ and $a^{j} \in Y$ such that $x a^{i} a^{j}=a^{j} x a^{i}$. Since $\operatorname{gcd}(j, p)=1$, we have $[x, a]=1$. Thus $a \in Z(G)$, so that $p|| Z(G) \mid$ and by the proof of Theorem 1.1, $p \leqslant|Z(G)|<\max \{m, n\}$.

LEMMA 4.4. Let $G$ be a non-Abelian finite group such that $|G / Z(G)|=4$. Then $G$ is not a $C(z, 2 z)$-group, where $z=|Z(G)|$.

Proof: Since $G$ is non-Abelian, $G / Z(G) \cong C_{2} \times C_{2}$. Thus there exist elements $a, b \in G$ such that

$$
G=Z(G) \cup a b Z(G) \cup a Z(G) \cup b Z(G) .
$$

Therefore $\langle a Z(G), b Z(G)\rangle$ is an elementary Abelian 2-group of order 4 . Thus $G=\langle a, b\rangle Z(G)$ and so $a b \neq b a$, since $G$ is not Abelian. Now consider the subsets $M=a Z(G) \cup b Z(G)$ and $N=a b Z(G)$. We have $x y \neq y x$ for all $x \in M$ and $y \in N$, since $a b \neq b a$. This shows that $G$ is not a $C(z, 2 z)$-group.

\section{REMARK 4.5.}

(1) Let $G$ be a $C(m, n)$-group. Then it is easy to see that $G$ is not a $C\left(t,\left|G \backslash C_{G}(a)\right|\right)$-group, where $a$ is any element of $G$ with $t \leqslant \mid C_{G}(a)$ $|Z(G)|$.

(2) If $G$ is a $C(m, n)$-group, then for any two natural numbers $m^{\prime}$ and $n^{\prime}$ such that $m \leqslant m^{\prime}$ and $n \leqslant n^{\prime}, G$ is also a $C\left(m^{\prime}, n^{\prime}\right)$-group.

Corollary 4.6. Let $G$ be a $C(1, n)$-group, where $5 \leqslant n \leqslant 9$. Then $G \cong S_{3}$, $D_{8}, Q_{8}, D_{10}, T, D_{12}$ or a non-Abelian group of order 16 whose centre is of order 4 .

Proof: By Remark 4.5(2), it is enough to consider only the case $n=9$. Suppose that $a$ is any non-central element of $G$. By Lemma 3.1 we have $\left|G \backslash C_{G}(a)\right| \leqslant 8$ and so $|G| \leqslant 16$. If $|G|=12$, then $G \cong A_{4}, D_{12}$ or $T$. The alternating group $A_{4}$ has an element whose centraliser has order 3 . Thus by Remark $4.5(1), A_{4}$ is not a $C(1,9)$-group. If $G \cong D_{12}$ or $G \cong T$, then the order of the centraliser of any element in $G$ is at least 4. Thus $G$ is a $C(1,9)$-group. If $|G|=14$, then $G \cong D_{14}$ and there exists $x \in D_{14}$ such that $\left|C_{G}(x)\right|=2$. By Remark $4.5(1), D_{14}$ is not a $C(1,9)$-group. Finally if $|G|=16$, 
then $|Z(G)|=2$ or 4 . If $|Z(G)|=4$, then for all $a \in G,\left|C_{G}(a)\right| \geqslant 8$. Thus $G$ is a $C(1,9)$-group. If $|Z(G)|=2$, then there exists an element $a$ in $G$ such that $\left|C_{G}(a)\right|=4$, so that by Remark $4.5(1), G$ is not a $C(1,9)$-group.

Corollary 4.7 . Let $G$ be a $C(2, n)$-group, where $4 \leqslant n \leqslant 8$. Then $G \cong S_{3}$, $Q_{8}, D_{8}$ or $D_{10}$.

Proof: By Remark 4.5(2), it is enough to consider only the case $n=8$. Since $G$ is non-Abelian, there exists an element $a$ in $G \backslash Z(G)$ such that $a^{2} \neq 1$. By Lemma $3.1,\left|G \backslash C_{G}(a)\right| \leqslant 7$, from which it follows that $|G| \leqslant 14$. If $|G|=12$, then $G$ contains centraliser of order 4 . Thus by Remark $4.5(1), G$ is not a $C(2,8)$-group. If $|G|=14$, then $G \cong D_{14}$, and it is not a $C(2,8)$-group since $D_{14}$ contains centralisers of order 2 .

LEmma 4.8. Let $G$ be a $C(3, n)$-group, where $3 \leqslant n \leqslant 7$. Then $G \cong S_{3}, D_{8}, Q_{8}$ or $D_{10}$.

Proof: By Remark 4.5(2), it is enough to consider only the case $n=7$. Since $G$ is non-Abelian, there exists non-central element $a$ in $G$ such that $a^{2} \neq 1$. Let $b \in G \backslash Z(G)$ be such that $b \neq a, a^{-1}$. Then by Lemma 3.1,

$$
\left|G \backslash C_{G}(a) \cup C_{G}\left(a^{-1}\right) \cup C_{G}(b)\right| \leqslant 6
$$

Hence $\left|G \backslash C_{G}(a) \cup C_{G}(b)\right| \leqslant 6$. Clearly $|G| \in\{8,10,12,14,16,20\}$. If $|G|=12$, then $G \cong A_{4}, D_{12}$ or $T$. As before $A_{4}$ is not a $C(3,7)$-group. For $D_{12}$, the subsets $M=\left\{b, b a^{3}, b a^{5}\right\}$ and $N=\left\{a, a^{2}, a^{4}, a^{5}, b a, b a^{2}, b a^{4}\right\}$ show that $D_{12}$ is not a $C(3,7)$ group. For $T$, the subsets $M=\left\{y, y x^{3}, x^{2} y\right\}$ and $N=\left\{x, x^{2}, x^{4}, x^{5}, y x, x y, y x^{2}\right\}$ show that $T$ is not a $C(3,7)$-group. For $|G|=14, G \cong D_{14}$ and there exists an element $x \in D_{14}$ such that $\left|C_{D_{14}}(x)\right|=7$, showing that $D_{14}$ is not a $C(3,7)$-group. If $|G|=16$, then $G$ has centralisers of order 8. By Remark $4.5(1), G$ is not a $C(3,7)$-group. Every non-Abelian group of order 20, has centralisers of order 4, and by Remark 4.5(1), is not a $C(3,7)$-group.

LEMmA 4.9. If $G$ is a $C(4,6)$-group and $Z(G) \neq 1$, then $G \cong Q_{8}$ or $D_{8}$.

Proof: By Lemma 3.3, $G /(Z(G))$ is an Abelian group and by Lemma 4.2, $\prod p \leqslant 5$. Thus $G$ is a $p$-group for $p \in\{2,3,5\}$. If $G$ is a 5 -group, then there ex$\prod_{\|\| G \mid} p$ ists an element $a$ in $G \backslash Z(G)$ whose order is 5 . Thus

$$
\left|G \backslash C_{G}(a) \cup C_{G}\left(a^{2}\right) \cup C_{G}\left(a^{3}\right) \cup C_{G}\left(a^{4}\right)\right| \leqslant 5 .
$$

Hence $\left|G \backslash C_{G}\left(a^{2}\right)\right| \leqslant 5$ and therefore $|G| \leqslant 10$, which is a contradiction. If $G$ is a 3-group, then by the proof of Theorem 1.1, $Z(G)=\langle z\rangle$, and there exists an element $a$ in $G \backslash Z(G)$ such that

$$
\left|G \backslash C_{G}(a) \cup C_{G}\left(a^{2}\right) \cup C_{G}(a z) \cup C_{G}\left(a z^{2}\right)\right| \leqslant 5
$$


Hence $\left|G \backslash C_{G}(a)\right| \leqslant 5$ and so $|G| \leqslant 10$, which is not possible. Therefore $G$ is a 2-group and by the proof of Theorem $1.1,|Z(G)|=2$ or 4 . Let $|Z(G)|=2$ and $Z(G)=\langle z\rangle$. Then there exists an element $a$ in $G \backslash Z(G)$ of order 4. Now we distinguish two cases:

CASE 1: $\quad a^{2} \notin Z(G)$. In this case

$$
\left|G \backslash C_{G}(a) \cup C_{G}\left(a^{2}\right) \cup C_{G}\left(a^{3}\right) \cup C_{G}(a z)\right| \leqslant 5 .
$$

Hence $\left|G \backslash C_{G}\left(a^{2}\right)\right| \leqslant 5$, so that $|G| \leqslant 10$, which cannot happen.

CASE 2: $\quad a^{2} \in Z(G)$. In this case there exists an element $b$ in $G \backslash\langle a\rangle$ such that

$$
\begin{gathered}
\left|G \backslash C_{G}(a) \cup C_{G}\left(a^{-1}\right) \cup C_{G}(b) \cup C_{G}\left(b a^{2}\right)\right| \leqslant 5 \text { and } \\
\left|G \backslash C_{G}(a) \cup C_{G}(b)\right| \leqslant 5 .
\end{gathered}
$$

Clearly $|G|=8$. Now suppose that $|Z(G)|=4$. Say, $Z(G)=\left\{1, z_{1}, z_{2}, z_{3}\right\}$. There exists an element $a$ in $G \backslash Z(G)$ of order 4 such that $a^{2} \neq z_{1}$, and

$$
\left|G \backslash C_{G}(a) \cup C_{G}\left(a^{2}\right) \cup C_{G}\left(a^{3}\right) \cup C_{G}\left(a z_{1}\right)\right| \leqslant 5 .
$$

Therefore $|G| \leqslant 10$, which is not possible again.

Lemma 4.10 . Let $G$ be a $C(4, n)$-group, where $4 \leqslant n \leqslant 6$. Then $G \cong S_{3}, Q_{8}$, $D_{8}$, or $D_{10}$.

Proof: By Remark 4.5(2), it is enough to consider only the case $n=6$. Let $a \in G \backslash Z(G)$. By Lemma 4.1, $|a| \in\{2,3,4,5,6,8,10,12\}$. Let $Z(G)=1$. We Distinguish three cases:

CASE 1. $|a| \geqslant 5$. In this case $\left|G \backslash C_{G}(a) \cup C_{G}\left(a^{2}\right) \cup C_{G}\left(a^{3}\right) \cup C_{G}\left(a^{4}\right)\right| \leqslant 5$ and $|G| \leqslant 10$.

CASE 2. $|a|=4$. For $b$ in $G \backslash\langle a\rangle$, we have $\left|G \backslash C_{G}(a) \cup C_{G}\left(a^{2}\right) \cup C_{G}\left(a^{3}\right) \cup C_{G}(b)\right| \leqslant 5$, from which it follows that $|G| \in\{8,10,12\}$. For $|G|=12$, then $G \cong A_{4}$, but the subsets

$$
\begin{aligned}
M & =\{(12)(34),(13)(24),(14)(23),(123)\} \text { and } \\
N & =\{(124),(142),(134),(143),(234),(243)\}
\end{aligned}
$$

show that $A_{4}$ is not a $C(4,6)$-group.

CASE 3. $|a| \in\{2,3\}$. In this case there exist elements $a$ and $b$ in $G$ of order 2 and 3 , respectively.

CASE 3(i). Suppose that there exists an element $c$ in $G \backslash\langle b\rangle$ of order 3. Then

$$
\left|G \backslash C_{G}(b) \cup C_{G}\left(b^{-1}\right) \cup C_{G}(c) \cup C_{G}\left(c^{-1}\right)\right| \leqslant 5,
$$

from which it follows that $|G|=10,12$ or 14 so that $G \cong D_{10}, A_{4}$ or $D_{14}$. The group $D_{14}$ has centraliser of order 7, and by Remark 4.5(1), it is not a $C(4,6)$-group. 
CASE 3(ii). Every $c$ in $G \backslash\langle b\rangle$ has order two. Let $a_{1}, a_{2}, a_{3}, a_{4}, a_{5}$ and $a_{6}$ be elements of order two. Then

$$
G=C_{G}\left(a_{1}\right) \cup C_{G}\left(a_{2}\right) \cup C_{G}\left(a_{3}\right) \cup C_{G}\left(a_{4}\right) \cup C_{G}\left(a_{5}\right) \cup C_{G}\left(a_{6}\right) \cup C_{G}(b) .
$$

Now by $\left[2\right.$, Theorem B], $|G| \leqslant 81$. But $|G|=2^{k} \cdot 3$ and hence $|G| \in\{6,12,24,48\}$. Since $A_{4}$ and $S_{4}$ are the only centreless groups of order 12 and 24 respectively which are not $C(4,6)$-groups, $|G| \neq 12$ or 24 .

Finally any centreless group of order 48 , has more than two elements of order 3 , so that $|G| \neq 48$. Now if $Z(G) \neq 1$, then by Lemma $4.9, G \cong Q_{8}$ or $D_{8}$, and the proof is complete.

Lemma 4.11. If $G$ is a $C(5,5)$-group, then $G \cong S_{3}, Q_{8}, D_{8}$ or $D_{10}$.

PROOF: A similar proof to that of Lemma 4.9, gives the result.

Proof of TheOREM 1.4: It follows easily from Lemmas 4.6-4.11.

\section{REFERENCES}

[1] A. Abdollahi, S. Akbari and H.R. Maimani, 'Non-commuting graph of a group', J. Algebra 298 (2006), 468-492.

[2] A. Abdollahi and S.M. Jafarian Amiri, 'On groups with an irredundant 7-cover', J. Pure Appl. Algebra (to appear).

[3] A. Abdollahi and A. Mohammadi Hassanabadi, 'Finite groups with certain number of elements pairwise generating a non-nilpotent subgroup', Bull. Iranian Math. Soc. 30 (2004), 1-20.

[4] E.A. Bertram, 'Some applications of graph theory to finite groups', Discrete Math. 44 (1983), 31-43.

[5] R.D. Blyth and D.J.S. Robinson, 'Insoluble groups with the rewriting property $P_{8}$ ', $J$. Pure Appl. Algebra 72 (1991), 251-263.

[6] R.A. Bryce, V. Fedri and L. Serena, 'Covering groups with subgroups', Bull. Austral. Math. Soc. 55 (1997), 469-476.

[7] G. Endimioni, 'Groupes finis satisfaisant la condition $(\mathcal{N}, n)$ ', C. R. Acad. Sci. Paris Ser. I Math. 319 (1994), 1245-1247.

[8] B. Huppert and N. Blackburn, Finite groups, III (Springer-Verlag, New York, 1982).

[9] B.H. Neumann, 'A problem of Paul Erdös on groups', J. Austral. Math. Soc. Ser. A 21 (1976), 467-472.

[10] B.H. Neumann, 'Ensuring commutativity of finite groups', J. Aust. Math. Soc. 71 (2001), 233-234.

[11] L. Pyber, 'The number of pairwise non-commuting elements and the index of the centre in a finite group', J. London Math. Soc. (2) 35 (1987), 287-295.

[12] D. Segal and A. Shalev, 'On groups with bounded conjugacy classes', Quart. J. Math. Oxford Ser. (2) 50 (1999), 505-516.

[13] J.G. Thompson, 'Nonsolvable finite groups all of whose local subgroups are soluble', Bull. Amer. Math. Soc. 74 (1968), 383-437. 
Department of Mathematics

University of Isfahan

Isfahan 81746-73441

Iran

e-mail: a.abdollahi@math.ui.ac.ir

a-azad@sci.ui.ac.ir

aamohaha@sci.ui.ac.ir

m.zarrin@math.ui.ac.ir 